\title{
Refrescando la memoria
}

Ahora que el ex presidente Alfredo Cristiani ha vuelto a la palestra política con mucha beligerancia y en algunos momentos - tanto en público como en privado- se expresa en términos poco amistosos sobre nuestra universidad, cabe traer a cuenta uno de los hechos en los que ciertamente él fue uno de los principales actores. En el marco de las tantas declaraciones que ha brindado recientemente y sobre todo pensando en una de sus afirmaciones puntuales más recientes - "el mundo abierto lo que requiere no es una dosis de ideología dogmática", dijo-, sería bueno que informara al país y al mundo sobre los datos que él conoce en relación con el caso de la matanza en la UCA ocurrida durante su mandato presidencial, el 16 de noviembre de 1989. Para ello, podemos ayudarle con algunos datos.

La historia de los ataques contra los jesuitas por parte de algunos sectores de la sociedad y de la FAES es ampliamente conocida. En abril de 1989, la campaña contra los sacerdotes de la UCA se recrudeció con campos pagados responsabilizados por el partido ARENA y la institución castrense. Después del asesinato del Dr. Roberto García Alvarado, Fiscal General de la República, el coronel Juan Orlando Zepeda -entonces comandante de la Primera Brigada de Infantería- afirmó que la Universidad Centroamericana era el centro de operaciones donde se había planificado dicho crimen. En seguida, la imprenta de la universidad fue objeto de un ataque dinamitero.

El 3 de julio de 1989, la Cruzada Pro Paz y Trabajo publicó una carta abierta al entonces presidente Cristiani en donde reclamaba la captura y el juicio sumario de, entre otros, los sacerdotes Ignacio Ellacuría y Segundo Montes, y los culpaba de "toda la destrucción de la infraestructura y de todos los viles y cobardes asesinatos que han cometido en nombre de la teología de la liberación". Al día siguiente, el entonces Viceministro de Seguridad Pública, coronel Inocente Orlando Montano, acusó a la UCA de valerse de espacios pagados "para tratar de desprestigiar a la Seguridad Pública y a la Fuerza Armada". Días después, siete bombas fueron colocadas en la imprenta de la UCA, que causaron cuantiosos daños materiales.

Pocas horas después de iniciada la ofensiva insurgente del 11 de noviembre de 1989, todas las estaciones radiofónicas debieron unirse en cadena nacional a la Radio Cuscatlán, emisora de la FAES. Bajo la dirección del Centro Nacional de Información (CNI), entre cuyos integrantes se incluía el jefe del Conjunto Cinco del Estado Mayor, coronel Carlos Armando Avilés Buitrago, se instaló un "micrófono abierto" mediante el cual las personas que llamaban por teléfono "denunciaban" a personalidades políticas de oposición, a dirigentes sindicales y eclesiales, a dirigentes del movimiento popular, etc., y los calificaban como "fachadas del FMLN". Estas declaraciones frecuentemente incitaban a la violencia contra dichas personas. Los ataques incluyeron también a los sacerdotes jesuitas, especialmente dirigidos contra el padre Ellacuría. "Ellacuría es un guerrillero. Que le corten la cabeza", "debemos sacar a Ellacuría para matarlo a escupidas", eran algunas de las expresiones que se escuchaban.

La primera noche de la ofensiva, el 11 de noviembre, la Fuerza Armada reportó que los policías nacionales que en la autopista sur prestaban 
seguridad en la Colonia Arce, fueron objeto de disparos desde la UCA. Además, el capitán Carlos Fernando Herrera Carranza, destacado en la Dirección Nacional de Inteligencia (DNI), reportó haber sido atacado desde la universidad cuando circulaba por la autopista sur.

El padre José María Tojeira declaró que el 12 de noviembre una patrulla de soldados entró en la UCA y se llevó un explosivo aparentemente dejado por elementos del FMLN que atravesaron el campus la noche anterior. Después de ese hecho, "un grupo de soldados estaba ubicado en la entrada del complejo universitario, registrando a todos los que entraban o salían y, desde el lunes 13 de noviembre, prohibiendo la entrada o salida de lodas las personas". Nunca se ha establecido quiénes fueron los soldados encargados de vigilar la entrada de la UCA, aunque la Fuerza Armada ha proporcionado varias listas de unidades apostadas entonces en esa zona.

El lunes 13 de noviembre se tomaron varias decisiones en una reunión realizada en el Estado Mayor que duró de las 14:00 hasta las 17:00 horas, con la presencia del jefe del Estado Mayor, el jefe de operaciones (Conjunto Tres), los directores de los cuerpos de seguridad, los comandantes de la zona metropolitana e, incluso, el coronel Guillermo Alfredo Benavides, director de la Escuela Militar. En dicha reunión, el entonces coronel René Emilio Ponce ordenó la formación del Comando de Seguridad del Complejo Militar que incluía el Ministerio de Defensa, el Estado Mayor, la Escuela Militar, la DNI, así como las colonias Arce y Palermo. Dentro de su perímetro también se encontraba la UCA. La sede del Comando se ubicó en la Escuela Militar "Capitán General Gerardo Barrios" y el coronel Benavides Moreno fue nombrado su jefe. El coronel Ponce también ordenó al coronel Oscar Alberto León Linares que desplazara su unidad de fuerzas especiales a la Escuela Militar, para que se agregara como refuerzo al Comando de Seguridad. Según los oficiales que asistieron a la referida reunión, no se trató el tema de la "presencia terrorista en la UCA".

Según consta en los registros del Batallón Atlacatl, a las 15:45 horas del 13 de noviembre salieron de su sede 110 elementos de tropa y cuatro oficiales en cuatro camiones, rumbo a San Salvador. Unos 47 hombres pertenecían a la unidad de comandos. El resto pertenecía a patrullas de otras compañías del Batallón. Aunque los integrantes de la unidad de comandos han sido identificados, nunca se identificaron a los otros soldados que llegaron a la capital con ellos. Según las declaraciones del coronel León Linares y del teniente José Ricardo Espinoza Guerra, el total de hombres era 135, aproximadamente. Sólo un miembro del Atlacatl que participó en una de las "patrullas adicionales" con los comandos, declaró judicialmente y admitió haber ido a la UCA en la madrugada del 16 de noviembre. Ese importante testigo es el subsargento Eduardo Antonio Córdova Monge.

Al final de la tarde del 13 de noviembre, el padre Ellacuría aterrizó en el aeropuerto de Comalapa y fue recibido por los padres Amando López y Miguel Francisco Estrada. Llegaron a la UCA pocos minutos antes del toque de queda. En el portón principal de la universidad, en la autopista sur, fueron detenidos por el retén de soldados apostado allí desde el día anterior. Sólo después que los soldados reconocieron al padre Ellacuría como el rector de la universidad los dejaron pasar. No se ha podido establecer qué unidad estaba vigilando la entrada de la UCA aquella tarde, ni si tenía instrucciones de informar de la llegada del padre Ellacuría. Sin embargo, consta en las actas de la Comisión de Investigación de Hechos Delictivos (CIHD) que miembros de la Policía de Hacienda apostados en la entonces llamada "Torre Democracia" manifestaron, un día después del crimen, que

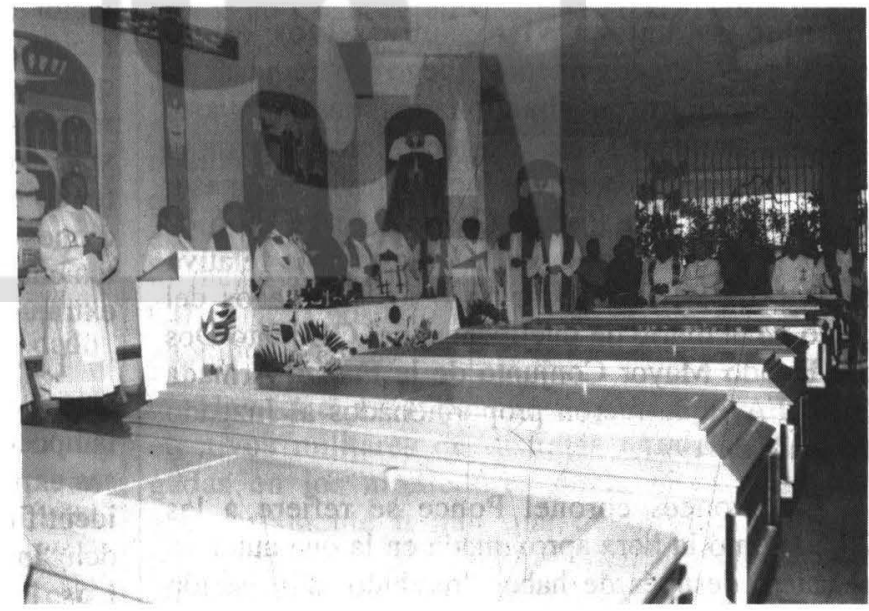


habían retenes de dicho cuerpo de seguridad en la entrada de la UCA. De ser cierto, habrían sido agentes de la Policía de Hacienda bajo el mando operacional de la DNI quienes, sin duda, habían estado en comunicación con esa instancia. Es significativo que nunca se haya podido determinar quién se encontraba allí, pese a toda la información conseguida sobre el despliegue de tropa en la zona.

El cateo a la residencia de los jesuitas es un aspecto capital por considerar en la secuencia de los hechos. La importancia del mismo se desprende del supuesto que fue un reconocimiento de terreno y la constatación del regreso del padre Ellacuría. Hay varios factores y muchas contradicciones sobre la hora del cateo, los motivos y la manera de llevarlo a cabo, que apuntan en esta dirección.

Al llegar a la capital, el oficial al mando de los comandos del Atlacatl, el teniente Espinoza Guerra, acompañado por su segundo, el subteniente Guevara Cerritos, se presentaron al Estado Mayor. Aunque las declaraciones varían sobre cuándo y dónde recibió la orden de efectuar un registro en la UCA, es claro que fue emitida desde el Estado Mayor. De hecho, el después general René Emilio Ponce admitió que la orden de cateo fue aprobada por sus superiores cuando manifestó que, "previa consulta al Señor Ministro del Ramo, General Rafael Humberto Larios López, quien a su vez habría consultado con el señor Presidente de la República y Comandante General de la Fuerza Armada,... aproximadamente a las 20:50 autorizó para que entraran a la UCA".

Por su parte, el ex presidente Cristiani reveló -hasta julio de 1990 - que autorizó el cateo "porque se habían visto subversivos entrar armados $\mathrm{y}$, efectivamente, luego de requisar el lugar, encontraron abandonados en un cuarto armas y uniformes que los guerrilleros dejaron al salir del recinto vestidos como civiles y pasar inadvertidos" (La Prensa Gráfica, 13 de julio de 1990, p. 69). Sin embargo, no consta nada relativo a las razones, la realización o los resultados del cateo en el diario de inteligencia del Conjunto Dos del Estado Mayor Conjunto de la Fuerza Armada (EMCFA), que fueron proporcionados al Juzgado en junio de 1991.

El entonces coronel Ponce se refiere a las 20:50 como la hora aproximada en la que autorizó el cateo, después de haber "recibido información de que terroristas habían penetrado (en la UCA) y además los mismos desde su interior hicieron fuego a personas militar desconociendo... el origen del informe". Mientras, el coronel Joaquín Arnoldo Cerna Flores manifestó, respecto al cateo, que éste fue ordenado entre las 19:30 y 19:45 horas del día 13. Las declaraciones de varios jesuitas y de los oficiales de la DNI establecen que el cateo empezó entre las 18:30 y 19:00 horas. Ante esta inconsistencia, Ponce cambió su versión al explicar que la orden original fue verificar la información recibida; sin embargo, la unidad del Atlacatl llamó por radio pidiendo autorización para entrar en la UCA. Esta versión se contradice con las declaraciones de Cerna Flores, Espinoza Guerra y los oficiales de la DNI antes referidas.

A pesar de la supuesta justificación del cateo, ninguno de los oficiales del Conjunto Dos que declaró en el proceso recordó haber recibido información sobre presencia guerrillera en la UCA, el día 13 de noviembre. El diario de inteligencia del Conjunto Dos, proporcionado al Juzgado después del período de prueba, el 27 de junio de 1991, tampoco incluye anotaciones al respecto. Los elementos de policía que daban seguridad a la Colonia Arce y que declararon en la causa, mencionaron haber sido atacados desde la UCA el día 11 de noviembre, no así el día 13. Ponce no pudo identificar la unidad que fue atacada el día 13.

La manera de realizarlo también sugiere que fue un reconocimiento del terreno y no un cateo para encontrar guerrilleros. Según los investigadores del New Scotland Yard, "el cateo del 13 de noviembre no parece haber sido muy minucioso, factor confirmado por comentarios en las declaraciones que describen el cateo como superficial y que únicamente algunos de los edificios fueron revisados, con frecuencia tan sólo mirando por las ventanas" Notaron que "el cateo pareció concentrarse en el Centro de Teologfa y en las habitaciones de los sacerdotes en particular". Es el teniente Espinoza Guerra quien, en su declaración extrajudicial, califica de "superficial" el cateo.

La presencia de un oficial de la DNI durante el cateo, el teniente Héctor Ulises Cuenca Ocampo, tampoco fue un hecho normal. Se han dado diversas explicaciones sobre ésta, pero de hecho no fue identificado plenamente antes de la publicación del "Informe Moakley", el 30 de abril de 1990. Los investigadores del New Scotland Yard 
encontraron sospechosa "la presencia del teniente Cuenca, de la DNI, cuya función era evidentemente obtener información y no hacer frente a terroristas. Este habría podido informar de la presencia del padre Ellacuría y de la ubicación y distribución de las habitaciones de los sacerdotes". Si fueron agentes de la Policía de Hacienda bajo el mando operacional de la DNI quienes avisaron del regreso del padre Ellacuría, el rol de la DNI se vuelve aún más sospechoso.

Un informe sobre el cateo, preparado por el teniente Cuenca Ocampo en diciembre de 1989, fue dirigido al jefe de operaciones -el ahora occiso capitán Herrera Carranza- quien lo pasó al subdirector de la DNI, el teniente coronel Nelson Iván Saldaña Araujo, quien a su vez lo turnó el 13 de diciembre de 1989 al entonces director de la DNI, el coronel Carlos Mauricio Guzmán Aguilar. Dicho informe no fue proporcionado a los encargados de la investigación del caso y sólo fue entregado al juez Ricardo Zamora por el general Ponce hasta el 18 de junio de 1991. De hecho, la presencia de un oficial de la DNI en el cateo no fue conocida por el juez hasta más de tres meses después de iniciada la causa.

Llevar a cabo un registro en la UCA era obviamente una cuestión delicada. Para realizarlo convenía consultar hasta los más altos niveles del poder civil y militar, tal como se hizo. Además, requería de un informe escrito amplio que diera constancia del mismo. El coronel León Linares proporcionó al coronel Ponce, el 8 de diciembre de 1989, un pequeño documento sobre el cateo que incluye sólo 35 nombres. Según este informe, el cateo concluyó sin novedad, "ya que no se encontró en dicho lugar a los D/T (delincuentes terroristas) que decía la fuente y que habían disparado a la patrulla de la FAES".

Otro aspecto por considerar es el hecho de que durante los días 14 y 15 de noviembre de 1989 , los comandos del Atlacatl se mantuvieron oficialmente- en la Escuela Militar bajo las órdenes del coronel Benavides quien, junto con su Plana Mayor, niega rotundamente haberles dado cualquier orden durante ese período.

No obstante, los registros de la sección de operaciones del Comando de Seguridad indican que, el día 14 de noviembre, "soldados del Atlacatl" detuvieron a una persona en las cercanías del cine Colonial. Detectives de la CIHD que realizaban una primera inspección un día después del crimen, interrogaron a Germán Orellana Vásquez, subsargento de la Policía Nacional, quien dijo "que vio pasar tanquetas y personal del Batallón Atlacatl mientras estaba de turno custodiando en la Torre Democracia, junto con Víctor Manuel Orellana Hernández". De igual manera, Gilberto Aguilar Flores, subsargento de la Policía de Hacienda, manifestó en primera instancia, el 17 de noviembre de 1989, a la CIHD que miembros del Batallón Atlacatl vigilaban el área de la residencial Guadalupe, aunque posteriormente - en su declaración de testigo del 8 de diciembre de 1989- manifestó no haber observado movilizaciones de tropa, ni luces de bengala, ni vehículos.

Acorde con las declaraciones extrajudiciales del teniente Espinoza Guerra y de varios elementos de su tropa, en la tarde del 15 de noviembre se encontraron desplegados alrededor de la UCA. Según los militares, ocupaban estas posiciones porque les habían notificado, a través de un supuesto informe de inteligencia, que los guerrilleros se dirigían hacia la universidad con sus heridos. Otros elementos que se tienen en el Juzgado no apoyan esta versión. El coronel Ponce dio otro dato completamente diferente, al manifestar que el Estado Mayor Conjunto de la Fuerza Armada había recibido, de la Policía Nacional, a las 16:30 horas del 15 de noviembre, un informe sobre una supuesta reunión de sindicalistas en la UCA a las 18:00 horas. El informe sobre esta reunión tampoco aparece en los registros del Conjunto Dos proporcionados al tribunal.

Los documentos presentados por el coronel Ponce no hacen referencia a la presencia de tropa del Atlacatl en la zona de la UCA el día 15 de noviembre. Elementos de otras unidades desplegadas en la zona niegan haber tenido conocimiento de la presencia de los soldados del Atlacatl, con excepción de algunos elementos policíacos apostados en la Torre Democracia que sólo lo mencionaron en una primera entrevista con la CIHD pero posteriormente, en sus declaraciones, ya no lo hicieron.

Hay que entender que habían numerosos efectivos militares de distintas unidades desplegadas en los alrededores de la UCA, quienes necesariamente tenían que darse cuenta de la presencia de las patrullas del Atlacatl. Habiéndose decretado el toque de queda a raíz de la ofensiva 
insurgente, desde las 18:00 a las 6:00 horas, y ante los rumores de un posible ataque del FMLN contra las principales instalaciones militares, era necesario identificar cualquier tropa en la zona. En la causa consta esta situación en la declaración de un miembro de la sección dos del Comando de Seguridad, el mayor Miguel Castillo González, y - de hecho- un guardia nacional destacado para vigilar la casa del Ministro de Economía, cerca del cine Colonial, que murio la noche del 15 de noviembre cuando unos soldados bajo el mando operacional de dicho Comando de Seguridad lo atacaron al no saber quién era.

Juan Antonio Navarro Artiga, agente de la Policía de Hacienda, declaró ante los detectives de la CIHD que cualquier tropa que hubiera pasado habría tenido que avisar por radio para que no le fueran a disparar. Tenían órdenes de disparar contra cualquier vehículo después de las 18:00 horas.

Entonces, el encubrimiento del crimen se extendió mucho más allá del Batallón Atlacatl y la Escuela Militar. Mucho antes de que la tropa Atlacatl fuera implicada públicamente en el crimen, los integrantes de todas las demás unidades en la zona sabían que tenían que decir que no se enteraron de nada, que no vieron nada y, concretamente, que no vieron a nadie del Batallón Atlacatl en las cercanías de la UCA entre el 15 y 16 de noviembre. De antemano, ya sabian que no debían saber nada.

A las 18:00 horas del 15 de noviembre se inició, en el Estado Mayor Conjunto de la FAES, una reunión con unos 24 militares de alta graduación. En ella estuvieron presentes el general Larios, el general Juan Rafael Bustillo, el coronel Ponce, el coronel Zepeda, el coronel Inocente Orlando Montano, el coronel Cerna Flores, el coronel Iván López y López, el coronel Carlos Armando Avilés, el coronel Juan Francisco Elena Fuentes, el coronel Benavides, el coronel León Linares, el coronel Juan Carlos Carrillo Schlenker, el coronel Heriberto Hernández, el coronel Dionisio Ismael Machuca, el coronel Guzmán Aguilar, el coronel Benjamín Eladio Canjura, el coronel Orlando Carranza, el coronel Francisco Raúl Arturo López, el coronel Roberto Esteban Santos, el coronel Ismar Francisco Roque, el teniente coronel Juan Vicente Eguizábal, el teniente coronel Juan Emilio Velasco Alfaro y el mayor Mauricio de Jesús Chávez Cáceres. La reunión duró hasta las $22: 40$ horas $y$, posterior- mente, se solicitó la presencia del presidente de la República como comandante en jefe de la FAES, para explicarle la situación y conseguir su autorización para cambiar el dispositivo militar.

De las 23:00 horas del 15 a las 00:30 horas del 16 de noviembre, según su declaración, el presidente Cristiani se encontró reunido con los integrantes del Alto Mando: el general Larios y los coroneles Zepeda, Montano y Ponce. El Lic. Cristiani, después de visitar el COCFA (Centro de Operaciones Conjuntas de la Fuerza Armada) acompañado de su Secretario Privado, el Lic. Arturo Tona, y esperar que se impartieran las nuevas órdenes, regresó a la residencia del Lic. Tona a las 02:30 horas, aproximadamente.

El Estado Mayor se mantenía al tanto de la situación nacional en el COCFA. Durante los días de la ofensiva, los integrantes del Alto Mando frecuentaron el COCFA, junto con los oficiales del Estado Mayor que normalmente estaban de turno. Además, oficiales de los Conjuntos Dos y Tres se turnaban para mantener una presencia permanente. También siempre estuvo presente un oficial de enlace de la Fuerza Aérea y otro de transmisiones.

Hasta ahora no ha sido posible averiguar sobre el papel o conocimiento del COCFA, relativo al operativo en la UCA durante la madrugada del día 16 de noviembre de 1989. En el proceso judicial, hasta antes del período de prueba de la fase plenaria, resultó imposible averiguar quién estaba a cargo del COCFA al momento del operativo. En su última declaración jurada rendida el 31 de mayo de 1991, durante el período de prueba de la fase plenaria, el general Ponce proporcionó - por primera vez- la siguiente información: "que de acuerdo a los registros de los servicios del Estado Mayor Conjunto de la Fuerza Armada, aparecen nombrados para el servicio en el COCFA, el señor Coronel Nelson Iván López y López como Jefe, el Teniente Raúl Antonio Mejía Chávez como Oficial de Servicio del Conjunto II, y el Mayor Oscar Joaquín Martínez Orellana como Oficial de Servicio del Conjunto III, desde las ocho horas del quince de noviembre de 1989 hasta las ocho horas del día dieciséis del mismo mes y año, aclarando el dicente, que los miembros del Alto Mando no realizaban turnos, sino que permanecían en el COCFA el tiempo que consideraban necesario según la situación..." 
A esas alturas, ya no existía oportunidad procesal para citar como testigos a los oficiales mencionados. El coronel López y López, señalado como el oficial superior a cargo del servicio en ese centro de operaciones, que debía tener el control de todas las operaciones en las circunstancias que entonces se vivían, declaró en la etapa de instrucción e incluso declaró sobre su participación en la investigación de la CIHD, pero no hizo referencia a su conocimiento sobre los hechos a partir de su condición de jefe a cargo del COCFA en el momento. Durante su declaración, el coronel López y López no fue interrogado acerca de sus responsabilidades en el COCFA, dado que no se sabía que las tenía. Habló sobre la reunión de jefes militares en el Estado Mayor Conjunto de la Fuerza Armada el 15 de noviembre de 1989, pero no mencionó que había pasado la noche al mando del COCFA, ni mucho menos lo que había sucedido durante su turno. Simplemente dijo que al finalizar la reunión de jefes militares "se quedó siempre en el interior del Estado Mayor Conjunto, pues se encontraban en un estado de emergencia".

El general Ponce había explicado anteriormente que no se organizaron turnos del Alto Mando para control del COCFA. Añadió que todos los miembros del Alto Mando permanecieron en el COCFA hasta como las 02:00 horas del 16 de noviembre, sin especificar quién quedó a cargo después y sin hacer tampoco mención de la presencia del Conjunto Uno en el COCFA. Por su parte, el coronel Joaquín Arnoldo Cerna Flores declaró que estuvo hasta la una o dos de la madrugada del 16 de noviembre en el COCFA, pero no recordó qué jefe de Conjunto lo relevó cuando se retiró a descansar. Según el coronel Ponce, durante los días de la ofensiva, los miembros del Alto Mando frecuentemente permanecían en el COCFA con las mismas facultades jerárquicas para dar órdenes y tomar decisiones operativas a nivel nacional.

La insistencia del general Ponce en aclarar que los miembros del Alto Mando no hicieron tumos en el COCFA puede haber sido la respuesta a lo declarado por el coronel Benjamín Eladio Canjura, el 2 de octubre de 1990. Según Canjura, "el Viceministro también puede impartir el tipo de orden antes relacionada en el aspecto operacional, pues se organizan turnos de trabajo, de seis u ocho horas, cuando los otros miembros se encuentren, por ejemplo descansando con el fin de mantener controlado el Centro de Operaciones Tácticas; que los turnos en mención los organiza el jefe del Estado Mayor, en época normal, pero en el mes de noviembre del año próximo pasado, durante la ofensiva, es posible que se hayan organizado los mismos de común acuerdo entre todos, pero más que todo por haber tomado parte en los turnos en mención, los señores Ministro y Viceministro de Defensa".

A su vez, el coronel Carlos Armando Avilés Buitrago, entonces jefe del Conjunto Cinco, declaró que en la madrugada del 16 de noviembre de 1989 recibió el informe de que se escuchaban fuertes detonaciones que provenían del sector del Complejo Militar, pero que no le especificaron los lugares de dónde provenían esas fuertes explosiones. Dijo "que tales informes, los daban las unidades que se encontraban operando en el complejo militar, y los que tenían que tomar decisiones operativas al respecto". No pudo precisar cuánto tiempo duraron esas fuertes explosiones, pero aseveró que: "al haberse escuchado esas explosiones, hubo preocupación extrema en el interior del Estado Mayor Conjunto; que existió asimismo preocupación de que los centros vitales de la Fuerza Armada, como serían el Estado Mayor Conjunto o el Ministerio de Defensa, fuesen atacados".

Avilés sostuvo que no sabía si a las unidades militares que informaron haber escuchado esas fuertes explosiones se les ordenó que ubicaran el lugar de dónde provenían. Puntualizó que desde que regresó al país, el 14 de noviembre, fue la primera vez que se temió que se efectuara algún ataque al Estado Mayor Conjunto o a otro centro vital de la Fuerza Armada. El mayor René Guillermo Contreras, jefe de contrainteligencia del Conjunto Dos, declaró que en la madrugada del 16 de noviembre de 1989 escuchó fuertes detonaciones y explosiones en las cercanías del Estado Mayor, ubicándolas por el sector de la UCA.

Según Avilés, el coronel Ponce, como jefe del EMCFA, tenía que haberse dado cuenta del peligro que representaban las explosiones escuchadas como parte de un posible ataque a los centros vitales de la Fuerza Armada, porque todos se encontraban en el interior del Estado Mayor. Avilés no recordó si el coronel Ponce se encontraba o no en el COCFA pero, si no lo estaba, "era obligación el informarle de dicha situación". 
En el mismo sentido, el coronel argentino José Luis García declaró que: “...si en el Estado Mayor Conjunto, dada la proximidad de los acontecimientos, se escucharon explosiones y otros disparos durante ese período con toque de queda, $y$ de no haberse recibido en ese comando información inmediata de lo que estaba ocurriendo, ese comando, de acuerdo a normas establecidas en los reglamentos militares, debió emplear sus propios medios para tener inmediata información de lo que ocurría".

En palabras del coronel Lanning Porter, miembro del grupo militar de Estados Unidos destinado como asesor del Conjunto Dos en la época, "la única reacción a los fuertes disparos y explosiones consistió, simplemente, en averiguar dónde ocurrían y qué era lo que sucedía".

Cabe hacer notar que la CIHD no indagó nada acerca de quién estaba encargado de o presente en el COCFA la noche del múltiple crimen. Cuando el juez de la causa recibió testimonio al respecto, sólo encontró contradicciones y vacíos. Hasta la última declaración del general Ponce, no había aparecido el nombre de un tan solo oficial que hubiera estado en el COCFA en el momento del crimen. Y ya no había oportunidad procesal para tomar declaración a los oficiales nombrados tan tarde. Además, no se había aclarado qué información recibieron o las medidas que tomaron respecto de los sucesos en la UCA. Sin embargo, quedó establecido que en el Estado Mayor se escuchaban perfectamente los disparos y las detonaciones en la UCA, y que los integrantes del Alto Mando estaban presentes en el Estado Mayor.
Nadie duda que la matanza del 16 de noviembre de 1989 en la UCA es uno de los capítulos más horrendos que registra la trágica historia de la última década en nuestro país. Estos hechos no hubieran sido posibles si en El Salvador no hubiera existido un amplio margen para el ejercicio criminal del poder, fuera del control del Organo Judicial y en abierta violación de la ley. La clara lección que se desprende de todo ello es que no basta con un funcionamiento formal de las instituciones republicanas para considerar que se está viviendo en un Estado de Derecho.

Traer a cuenta ahora todos los elementos que aparecen en este comentario tiene dos propósitos. Por un lado, advertir que - pese a todo lo sucedido desde esa trágica fecha hasta este momentosiguen existiendo casos y situaciones reveladoras de una situación de impunidad que todavía no ha sido superada. El segundo, pedirle al ex presidente Cristiani que contribuya en algo a superar esa grave lacra que continúa poniendo en tela de juicio cualquier avance del cual quiera presumir. Para ello, podría comenzar por informar sobre lo que sucedió durante esa noche cuando él se encontraba en la sede del Estado Mayor Conjunto de la FAES y se escucharon las fuertes detonaciones en la UCA. Se lo pedimos ahora que está hablando nuevamente, que manifiesta - tal como afirmó a un periódico nacional- sentirse "completamente tranquilo" y que su "conciencia está muy clara".

Instituto de Derechos Humanos de la Universidad Centroamericana "José Simeón Cañas" 